\title{
Pseudotumor Cerebri: Categorical Disease or Spectrum of Disease
}

\author{
M. Sami Walid ${ }^{\mathrm{a}, \mathrm{e}}$, Mazen Sanoufa ${ }^{\mathrm{a}}$, Joe Sam Robinson, III ${ }^{\mathrm{b}}$, Maragaret C. Boltja ${ }^{\mathrm{c}}$, \\ Joe Sam Robinson, Jr ${ }^{\mathrm{d}}$
}

\begin{abstract}
Background: Idiopathic intracranial hypertension (aka. pseudotumor cerebri) is a rare neurological disease of complex etiology that is more common in obese females. In this study, we investigate the main characteristics of our patient cohort with the diagnosis of idiopathic intracranial hypertension.
\end{abstract}

Methods: The charts of pseudotumor cerebri patients that underwent treatment during the past five years at our institution in Central Georgia were retrospectively reviewed. We collected data on age, ethnicity, obesity, presence of papilledema, comorbidities, radiographic findings, laboratory findings and treatment. The modified Dandy criteria for the diagnosis of pseudotumor cerebri were applied on the patients.

Results: The modified Dandy criteria applied to 23 patients with diagnosis of pseudotumor cerebri. The mean age was 35 and the range 15 to 62 years old. The average BMI was 39 and the range 22 to $62 \mathrm{~kg} / \mathrm{m}^{2}$. All of the 23 patients were females. Fourteen patients $(63.6 \%)$ had papilledema related to the diagnosis of idiopathic intracranial hypertension. Twelve $(85.7 \%)$ patients of those with papilledema (14) were obese compared with five $(55.6 \%)$ patients of those without papilledema (9). We had only two patients who had the full complex of symptoms including headaches, elevated opening pressure, papilledema and small cerebral ventricles. Six of seven diabetic patients had papilledema. Four of seven patients with arterial hypertension had papilledema. Two of three patients with chronic renal disease had papilledema. Four patients had lowlying cerebellar tonsils including three with papilledema.

Manuscript accepted for publication February 22, 2011

${ }^{a}$ Medical Center of Central Georgia, Macon, GA, USA

${ }^{\mathrm{b}}$ Mercer University School of Medicine, Macon, GA, USA

${ }^{c}$ Neurology Associates, Macon, GA, USA

${ }^{\mathrm{d}}$ Georgia Neurosurgical Institute, Macon, GA, USA

${ }^{\mathrm{e} C}$ Corresponding author: M. Sami Walid, Email: mswalid@yahoo.com

doi:10.4021/jnr103e
Conclusions: A cohort of patients with pseudotumor cerebri diagnosis presents a spectrum of the disease with increasing severity. A significant percentage of these patients have metabolic comorbidities or intracranial malformations that may explain the symptoms and should be thoroughly investigated.

Keywords: Idiopathic intracranial hypertension; Pseudotumor cerebri; Papilledema; Small cerebral ventricles

\section{Introduction}

Idiopathic intracranial hypertension is a rare neurological disease of complex etiology that is more common in obese females. Idiopathic intracranial hypertension, aka. pseudotumor cerebri (PTC), is characterized by increased intracranial pressure and papilledema in the absence of other neurologic localizing signs. Headaches and visual disturbances are the most common manifestations of the disease. The incidence of PTC in the general population is around $1: 100,000$, whereas in obese women aged 15 - 44 it is more common, at $10-20: 100,000$ [1]. PTC also occurs in approximately one in 1000 pregnancies [2].

In this study, we investigate the main characteristics of our patient cohort with the diagnosis of idiopathic intracranial hypertension.

\section{Materials and Methods}

The charts of pseudotumor cerebri patients that underwent treatment during the past five years at our institution in Central Georgia were retrospectively reviewed. We collected data on age, ethnicity, obesity (BMI $\geq 30 \mathrm{~kg} / \mathrm{m}^{2}$ ), presence of papilledema, comorbidities, radiographic findings, laboratory findings and treatment.

The Dandy criteria $[3,4]$ for the diagnosis of pseudotumor cerebri were applied on the patients: (1) Signs and symptoms of increased intracranial pressure (headache, nausea, vomiting, transient obscurations of vision, papilledema); 


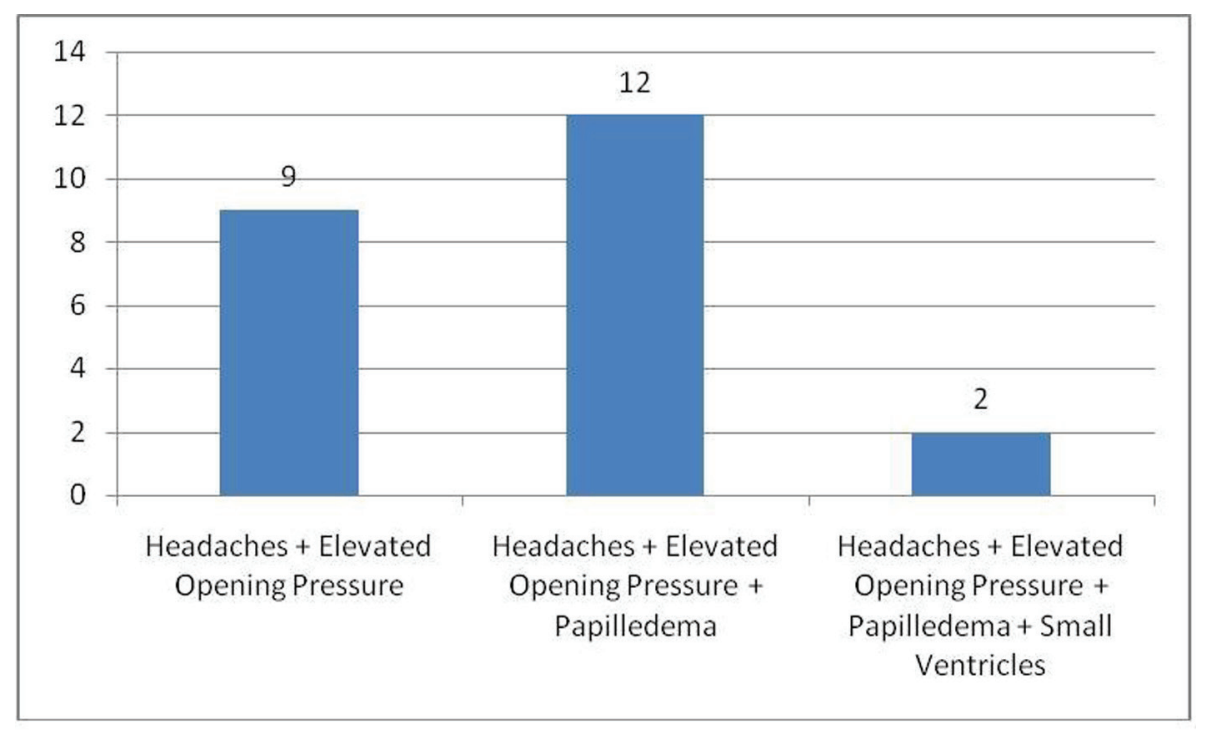

Figure 1. Distribution of patients by headaches, opening pressure, papilledema and ventricle size.

(2) Normal neurological exam, except for possible 6th nerve paresis; (3) Elevated cerebrospinal fluid pressure ( $>250 \mathrm{~mm}$ H2O) with normal constituents; (4) Neuroimaging excluding a mass lesion or hydrocephalus.

\section{Results}

The modified Dandy criteria applied to 23 patients with diagnosis of PTC. The mean age was 35 and the range 15 to 62 years old. The average BMI was 39 and the range 22 to $62 \mathrm{~kg} / \mathrm{m}^{2}$. All of the 23 patients were females. Eleven were Afro-Americans and seven Caucasians and the rest of mixed race. Six patients reported being smokers. All of the 23 patients complained of headaches and twenty complained of blurred vision at the time of their presentation. Fourteen patients $(63.6 \%)$ had papilledema related to the diagnosis of idiopathic intracranial hypertension (Fig. 1).

Twelve (85.7\%) patients of those with papilledema (14) were obese compared with five $(55.6 \%)$ patients of those without papilledema (9). Only two patients had the full complex of symptoms including headaches, elevated opening pressure, papilledema and small cerebral ventricles (Fig. 1). One patient developed the symptoms when she gained more than 25 pounds after starting a course of SSRI (Paxil) for depression. Fourteen patients had ventriculoperitoneal shunt (Fig. 2), nine recurrent lumbar punctures, and fourteen were treated with acetazolamide. One patient developed neutropenia as an adverse effect of acetazolamide use. Optic nerve fenestration was required to prevent loss of vision in two patients.

Six of seven diabetic patients had papilledema. Four of seven patients with arterial hypertension had papilledema.
Two of three patients with chronic renal disease had papilledema. Four patients had low-lying cerebellar tonsils including three with papilledema. Twelve patients had high red cell distribution width (RDW); ten of them with papilledema (Fig. 3).

\section{Discussion}

PTC typically affects obese females of reproductive age. An

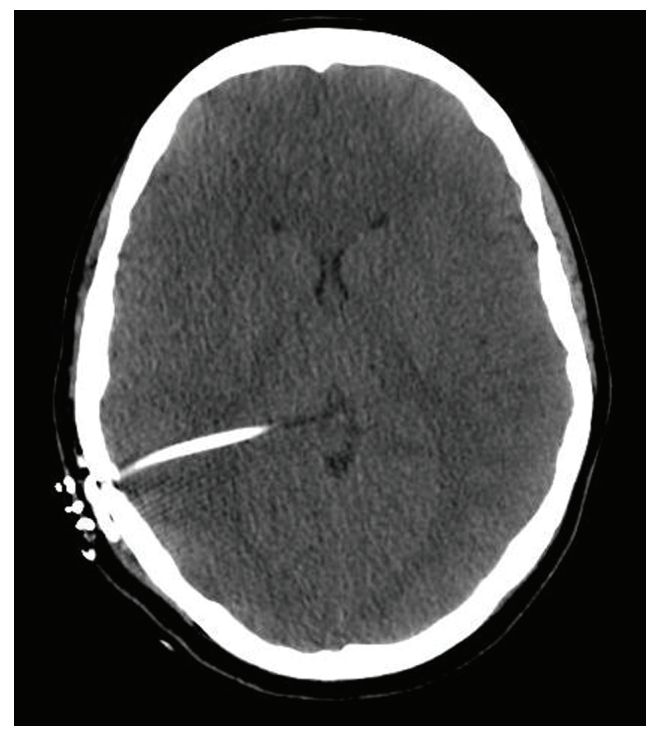

Figure 2. Computed tomography image of the brain showing small ventricles and shunt. 


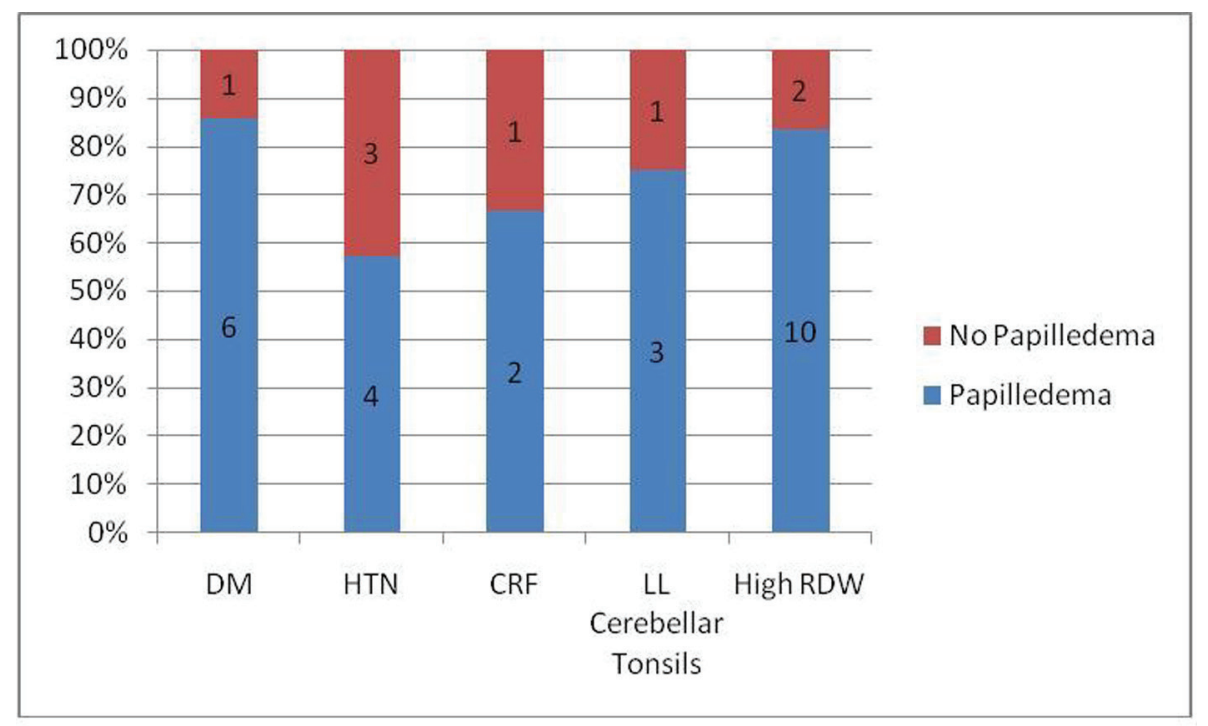

Figure 3. Percentage of papilledema in PTC patients with comorbid conditions. DM: Diabetes mellitus; HTN: Hypertension; CRF: Chronic renal failure; LL cerebellar tonsils: Low-lying cerebellar tonsils.

untypical patient may be a man, a child or a thin person in which case an organic cause of increased intracranial pressure should be first sought [5]. PTC's etiology is not well understood. Many theories exist on PTC's pathomechanism. The mainstream of clinical research link this disease to the water retention effect of estrogens on the central nervous system $[5,6]$. However, this theory is contradicted by reports of PTC occurrence in males or in females with Turner's syndrome $[7,8]$. Less popular theories talk about disturbances in the hypothalamic-hypophyseal-ovarian axis in response to stressful triggers, an allergic Jarisch-Herxheimer-type reaction to some undetermined infectious or noninfectious stimuli or theories that incriminate thrombophilia and/or hypofibrinolysis that lead to microthrombi in the choroid plexus leading to decreased drainage of the cerebrospinal fluid (CSF) and increased intracranial pressure [9-11]. The last theory seems to confuse intracranial hypertension due to increased CSF pressure with increased intracranial pres-

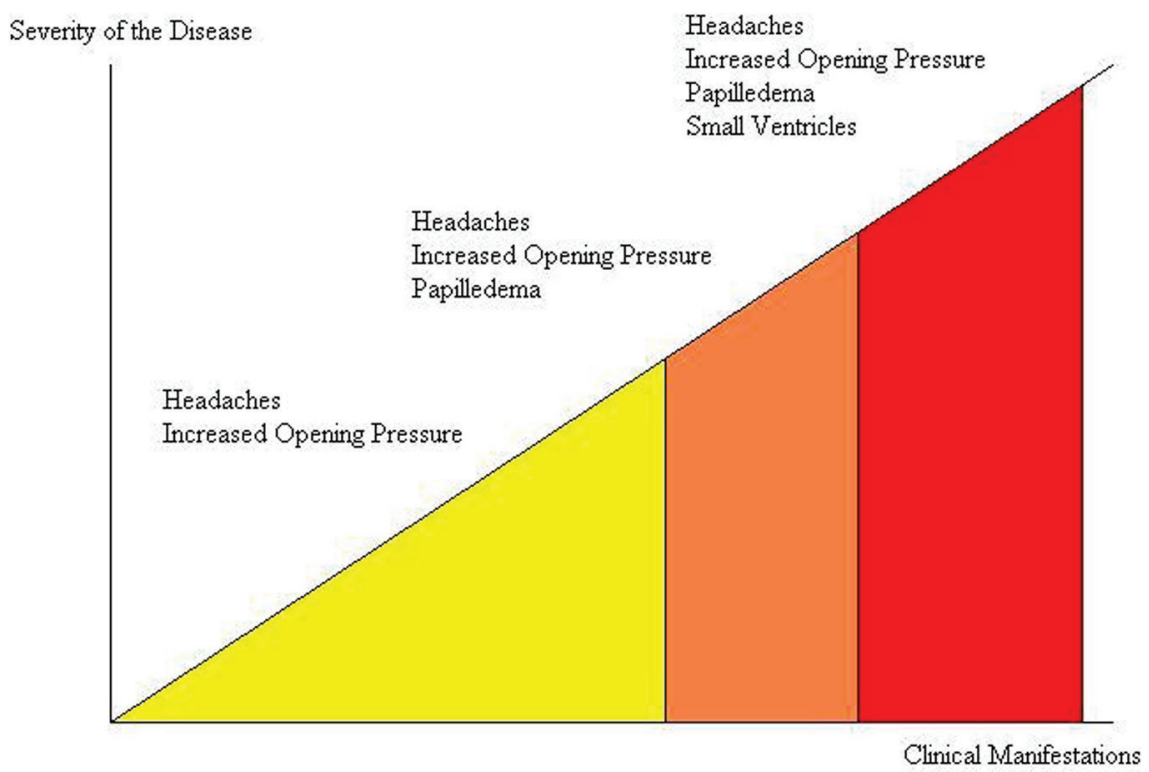

Figure 4. Graph depicting the severity of disease by signs and symptoms. 
sure due to increased volume of brain tissue. The definition of idiopathic intracranial hypertension requires the absence of any focal lesion that may cause an increase in intracranial pressure or ventriculomegaly. Thus, increased intracranial pressure that is associated with ventriculomegaly is called hydrocephalus and not pseudotumor cerebri, aka. idiopathic intracranial hypertension, implying that no apparent focal cause of increased intracranial pressure can be identified. Hydrocephalus with increasing intracranial pressure implies a problem with the production or drainage of the CSF. However, in pseudotumor cerebri, the brain tissue itself is swollen due to intracellular or intercellular water retention. This increases the opening pressure of lumbar puncture by contiguity and manifests itself ophthalmologically with papilledema. Additionally, the posterior lobe of the pituitary gland located in the sella turcica may be affected by the increased intracranial pressure causing increased secretion of the antiduiretic hormone which may further exacerbate the situation.

In this paper we presented a case series of pseudotumor cerebri patients from a single neurosurgical provider showing that the disease is not a categorical diagnosis with Yes/No designation. A cohort of patients with PTC diagnosis forms a spectrum of the disease with increasing severity (Fig. 4). Thus, female obese patients with headaches and elevated opening pressure may be viewed as transitional patients between normal and PTC patients. Increased opening pressure without papilledema may be a situational finding due to stress, anxiety or hypertension. More severely affected patients are patients with headaches, increase opening pressure and papilledema, an ophthalmologic sign that objectively reflects increased intracranial pressure. The most severely affected group of patients is those with headaches, increase opening pressure, papilledema, and decreased ventricle size due to increased parenchymal tissue volume. We had only two patients who had the full range of symptoms including small cerebral ventricles. The moderate and severe groups of PTC patients most often require surgical intervention with lumbar peritoneal shunt placement to avert the risk of vision loss. Optic nerve sheath fenestration is used in advanced cases to reduce the pressure on the optic disc [12].

The term "benign intracranial hypertension" was first used by Foley [13] then was advocated by Weisberg [14] and even used by more recent authors [15-17]. This designation should be avoided, however, because the condition may be associated with severe debilitating visual loss in as many as $25 \%$ of patients $[18,19]$, and therefore it is not always "benign". Instead, experts today advocate the use of the more accurate term "idiopathic intracranial hypertension". However, even this description is not accurate because most of these patients are obese females and therefore the cause is not totally unknown (i.e. not idiopathic).

Pseudotumor cerebri patients should be scrupulously interviewed and investigated to reveal any physiologic devia- tions or intracranial malformations that may explain weight gain, water retention and symptoms of increased intracranial pressure. If manageable metabolic or organic aberrances are discovered then the trigger of increased intracranial pressure should be treated before applying the diagnosis of "Idiopathic Intracranial Hypertension". Inferior tonsillar displacement, for example, may be detected on magnetic resonance imaging in a significant percentage of PTC patients possibly representing a secondary form of PTC which may benefit from correction of ITD to restore normal intracranial pressure [20]. Another simple example of secondary etiology is the development of PTC as a rare complication of iron deficiency anemia [21]. Increased RDW may be the earliest evidence of the development of an iron-deficient erythropoiesis in the peripheral blood smear.

The diagnosis of pseudotumor cerebri can be difficult in atypical cases where some elements of the Dandy criteria are missing, for example, how to differentiate functional headaches with situational elevation of opening pressure from atypical pseudotumor cerebri without papilledema [22]. In light of this, it is useful to quote Dr. F. H. Sklar's first paragraph in the introduction of his chapter on Pseudotumor Cerebri in Neurosurgery, 2nd Edition, 1996, "Unfortunately, the term has been used to describe patients with a potpourri of clinical conditions. Unrelated disease mechanisms may share similar clinical presentations. Accordingly, pseudotumor cerebri likely represents multiple and different disease entities. This nonspecificity of pathophysiology has most certainly been a major contributing factor to confusion and controversy associated with this topic." [23].

\section{References}

1. Ivancic R, Pfadenhauer K. Pseudotumor cerebri after hormonal emergency contraception. Eur Neurol 2004;52(2):120.

2. Katz VL, Peterson R, Cefalo RC. Pseudotumor cerebri and pregnancy. Am J Perinatol 1989;6(4):442-445.

3. Smith JL. Whence pseudotumor cerebri? J Clin Neuroophthalmol 1985;5(1):55-56.

4. Wall M. Idiopathic intracranial hypertension. Neurol Clin 1991;9(1):73-95.

5. Bagga R, Jain V, Das CP, Gupta KR, Gopalan S, Malhotra S. Choice of therapy and mode of delivery in idiopathic intracranial hypertension during pregnancy. MedGenMed 2005;7(4):42.

6. Donaldson JO, Horak E. Cerebrospinal fluid oestrone in pseudotumour cerebri. J Neurol Neurosurg Psychiatry 1982;45(8):734-736.

7. Digre KB, Corbett JJ. Pseudotumor cerebri in men. Arch Neurol 1988;45(8):866-872.

8. Donaldson JO, Binstock ML. Pseudotumor cerebri in 
an obese woman with Turner syndrome. Neurology 1981;31(6):758-760.

9. Powell JL. Pseudotumor cerebri and pregnancy. Obstet Gynecol 1972;40(5):713-718.

10. Barber SG, Garvan N. Is "benign intracranial hypertension" really benign? J Neurol Neurosurg Psychiatry 1980;43(2):136-138.

11. Finsterer J, Kuntscher D, Brunner S, Krugluger W. Pseudotumor cerebri from sinus venous thrombosis, associated with polycystic ovary syndrome and hereditary hypercoagulability. Gynecol Endocrinol 2007;23(3):179-182.

12. Corbett JJ, Thompson HS. The rational management of idiopathic intracranial hypertension. Arch Neurol 1989;46(10):1049-1051.

13. Foley J. Benign forms of intracranial hypertension; toxic and otitic hydrocephalus. Brain 1955;78(1):1-41.

14. Weisberg LA. Benign intracranial hypertension. Medicine (Baltimore) 1975;54(3):197-207.

15. Greitz D, Hannerz J, Rahn T, Bolander H, Ericsson A. MR imaging of cerebrospinal fluid dynamics in health and disease. On the vascular pathogenesis of communicating hydrocephalus and benign intracranial hypertension. Acta Radiol 1994;35(3):204-211.

16. Malozowski S, Tanner LA, Wysowski DK, Fleming GA, Stadel BV. Benign intracranial hypertension in children with growth hormone deficiency treated with growth hormone. J Pediatr 1995;126(6):996-999.
17. Schwarz S, Husstedt IW, Georgiadis D, Reichelt D, Zidek W. Benign intracranial hypertension in an HIVinfected patient: headache as the only presenting sign. AIDS 1995;9(6):657-658.

18. Corbett JJ, Savino PJ, Thompson HS, Kansu T, Schatz NJ, Orr LS, Hopson D. Visual loss in pseudotumor cerebri. Follow-up of 57 patients from five to 41 years and a profile of 14 patients with permanent severe visual loss. Arch Neurol 1982;39(8):461-474.

19. Lessell S, Rosman NP. Permanent visual impairment in childhood pseudotumor cerebri. Arch Neurol 1986;43(8):801-804.

20. Banik R, Lin D, Miller NR. Prevalence of Chiari I malformation and cerebellar ectopia in patients with pseudotumor cerebri. J Neurol Sci 2006;247(1):71-75.

21. Tugal O, Jacobson R, Berezin S, Foreman S, Brudnicki A, Godine L, Davidian MM, et al. Recurrent benign intracranial hypertension due to iron deficiency anemia. Case report and review of the literature. Am J Pediatr Hematol Oncol 1994;16(3):266-270.

22. Digre KB, Nakamoto BK, Warner JE, Langeberg WJ, Baggaley SK, Katz BJ. A comparison of idiopathic intracranial hypertension with and without papilledema. Headache 2009;49(2):185-193.

23. Sklar FH. Pseudotumor Cerebri. In: Neurosurgery. Wilkins RH, Rengachary SS (Eds.). New York : McGraw-Hill, Health Professions Division, c1996. 